\title{
Comparação entre dois protocolos de tratamento de ceratoconjuntivite seca experimentalmente induzida em coelhos
}

\author{
[Comparison of two treatment protocols of experimentally induced \\ keratoconjunctivitis sicca in rabbits] \\ D.A. Motta $^{1}$, L. Yamasaki ${ }^{2}$, O.C. Sanches ${ }^{2}$, R. Giuffrida ${ }^{3}$, E.R. Cândido ${ }^{3}$, C.S.G. Pereira ${ }^{3}$, J.E. Góes ${ }^{3}$, \\ T.L.A. Rocha ${ }^{3}$, D. A. Silva ${ }^{3}$, M.C.A. Silva ${ }^{4}$, K.M. Basso ${ }^{4}$, S.F. Andrade ${ }^{5 *}$ \\ ${ }^{1}$ Aluna de pós-graduação - Unoeste- Presidente Prudente, SP \\ ${ }^{2}$ Departamento de Anatomia Patológica - Unoeste - Presidente Prudente, SP \\ ${ }^{3}$ Faculdade de Medicina Veterinária - Unoeste - Presidente Prudente, SP \\ ${ }^{4}$ Hospital Veterinário - Unoeste - Presidente Prudente, SP \\ ${ }^{5}$ Departamento de Clínica Médica de Pequenos Animais - Unoeste - Presidente Prudente, SP
}

\begin{abstract}
RESUMO
O objetivo deste estudo foi avaliar e comparar a eficácia de dois protocolos de tratamento de ceratoconjuntivite seca (CCS) experimentalmente induzida em coelhos: uma formulação oftálmica tópica composta por álcool polivinílico $1,4 \%$, adicionado com acetilcisteína $10 \%$ e pilocarpina $1 \%$ (AAP), e outro protocolo com o uso do óleo de semente de linhaça (OL) tópico em forma de colírio, durante 12 semanas. Foram utilizados 15 coelhos machos, adultos, da raça Nova Zelândia, alocados aleatoriamente em três grupos: grupo C (controle), grupo AAP (formulação oftálmica) e grupo L (OL tópica). Os animais foram avaliados semanalmente pelo teste lacrimal de Schirmer, teste de fluoresceína e teste de Rosa Bengala; uma vez por mês, pelo exame de citologia esfoliativa ocular; ao final do experimento, pela análise histopatológica da córnea e conjuntiva. Os resultados demonstraram que houve um aumento maior na produção lacrimal quando utilizada a formulação oftálmica, e uma resolução mais rápida das úlceras de córnea, bem como diminuição no número de células desvitalizadas quando utilizado o óleo de semente de linhaça, além de aumento no número de células caliciformes em ambos os grupos de tratamento. A associação desses dois protocolos pode ser no futuro uma alternativa no tratamento da CCS.
\end{abstract}

Palavras-chave: ceratoconjuntivite seca, álcool polivinílico 1,4\%, acetilcisteína 10\%, pilocarpina $1 \%$, óleo de linhaça

\begin{abstract}
The objective of this study was to evaluate and compare the effectiveness of two treatment protocol of experimentally induced keratoconjunctivitis sicca (KCS) in rabbits, a topical ophthalmic formulation composed by $1.4 \%$ povinilic alcohol added with $10 \%$ acetylcysteine and $1 \%$ pilocarpine (AAP) and another protocol with the topical use of the linseed seed oil (LO) in eye drop form for 12 weeks. Fifteen male New Zealand white rabbits were aleatory allocated in 3 groups: Group C (Control), Group AAP (ophthalmic formulation) and Group L (LO topical). The animals were evaluated weekly using the Schirmer's tear test, fluorescein test and Rose Bengal test monthly for ocular cytology, and at the end of the experiment for histopathological analysis of cornea and conjunctive. The results demonstrated that there was a larger increase in the tear production when the ophthalmic formulation was used and a faster rapid resolution of corneal ulcers and decrease in the number of devitalized cells when linseed seed oil was used, besides an increase in the number of caliciform cells in both treatment groups. The association of those two protocols can be a future alternative in the treatment of KCS.
\end{abstract}

Keywords: keratoconjunctivitis sicca, $1.4 \%$ povinilic alcohol, $10 \%$ acetylcysteine, $1 \%$ pilocarpine, linseed oil

Recebido em 26 de junho de 2012

Aceito em 20 de maio de 2013

*Autor para correspondência (correspondig author)

E-mail: silviafranco@unoeste.br 


\section{INTRODUÇÃO}

A ceratoconjuntivite seca (CCS) ou olho seco é uma das afecções oftálmicas mais comumente encontradas nos animais da espécie canina e nos seres humanos, caracterizando-se pela deficiência do componente aquoso do filme lacrimal pré-corneano (FLP) ou pela modificação da qualidade ou, ainda, pela diminuição da estabilidade da lágrima, o que leva a um comprometimento no deslizamento das pálpebras, afetando principalmente a córnea e a conjuntiva bulbar (Miller, 2008; Stevenson et al., 2012).

Os principais sinais clínicos são secreção mucoide a mucopurulenta, perda de brilho e pigmentação da córnea, hiperemia conjuntival, desconforto ocular, prurido, vascularização da córnea, ulcerações superficiais a profundas, podendo evoluir para perda progressiva da visão (Carter e Colitz, 2002). O diagnóstico baseia-se principalmente nos sinais clínicos e nos resultados obtidos com os exames oftálmicos, tais como teste lacrimal de Schirmer (TLS), teste de Rosa Bengala (TRB), teste de fluoresceína (TF), tempo de ruptura do filme lacrimal (TRFL), teste de lisamina verde, além de citologia esfoliativa (CE) (Maggs, 2008; Miller, 2008).

Existem vários tipos de tratamento para a CCS, sendo mais utilizados atualmente na medicina veterinária os imunossupressores tópicos, tais como ciclosporina, tacrolimus e pimecrolimus (Izci et al., 2002; Berdoulay et al., 2005; Ofri et $a l$. , 2009). Em razão do alto custo desses medicamentos, uma alternativa mais barata, principalmente para proprietários carentes, consiste em fazer uso de associações de substâncias em formulações tópicas, como as que utilizam álcool polivinílico (substituto aquoso da lágrima, que estabiliza a película lacrimal pré-corneal e impede a sua ruptura) associado à acetilcisteína 10\% (agente anticolagenase, devido à sua ação mucolítica) e pilocarpina $1 \%$ (pelo seu efeito lacrimoestimulante). A proporção usada na formulação oftálmica (substituto lacrimal + acetilcisteína 10\% + pilocarpina 1 a $2 \%$ ) varia segundo alguns autores, respectivamente, de $3,5 \mathrm{~mL} / 3,5 \mathrm{~mL} / 1 \mathrm{~mL}$ (Andrade, 2008), $7 \mathrm{~cm}^{3}$ (cc) $/ 7 \mathrm{cc} / 2 \mathrm{cc}$ (Kirk, 1984; Severin, 1995) e $15 \mathrm{~mL} / 2-4 \mathrm{~mL} / 2 \mathrm{~mL}$ acrescidos de $2 \mathrm{~mL}$ de cloranfenicol injetável a 2\% (Pickett, 2001). Além disso, anti-inflamatórios esteroidais e não esteroidais e antibióticos podem ser associados à terapia como adjuvantes (Carter e Colitz, 2002; Stevenson et al., 2012).

Outra forma de tratamento para a CCS, que vem se destacando em estudos recentes, é a utilização de ácidos graxos essenciais (AGE), ômega 3 ( $\omega$ $3)$ e ômega $6(\omega-6)$, principalmente no tratamento de pacientes portadores de diversas formas de deficiência lacrimal, como na Síndrome de Sjögren humana, tanto em suplementação oral (Barabino et al., 2003; Mavragani et al., 2006; Pinheiro Jr. et al., 2007; Roncone et al., 2010; Wojtowicz et al., 2011), como em experimentos por via tópica em ratos (Rashid et al., 2008) e por via tópica e oral em coelhos (Neves et al., 2013). Os principais efeitos terapêuticos do $\omega-3$ e $\omega-6$ são imunomodulador e anti-inflamatório. A semente de linhaça é uma planta da família Linaceae, chamada de Linum usitatissimun, composta, em média, por $57 \%$ de ácidos graxos ômega 3, $16 \%$ de ômega $6,18 \%$ de ácido graxo monoinsaturado e somente $9 \%$ de ácidos graxos insaturados, sendo sua relação $\omega-3$ e $\omega-6$ de 1:3 considerada muito próxima do ideal (Oomah, 2001; HassanZadeh et al., 2008).

O objetivo deste trabalho foi comparar a eficácia de duas formulações tópicas, uma composta por substitutos da lágrima adicionada de substância lacrimoestimulante e anticolagenase, e outra composta por óleo de linhaça, no tratamento da CCS induzida experimentalmente em coelhos.

\section{MATERIAL E MÉTODOS}

$\mathrm{O}$ experimento foi aprovado (protocolo $\mathrm{n}^{\circ} 166$ ) pela Comissão de Ética no Uso de Animais (Ceua) da Universidade do Oeste Paulista (Unoeste). Foram utilizados 15 coelhos (Oryctolagus cuniculus) brancos, machos, da raça Nova Zelândia, não castrados, adultos, e com peso variando entre 2 e $3 \mathrm{~kg}$. Estes animais foram mantidos em gaiolas metálicas individuais suspensas, com água e ração ad libitum.

Para a indução da CCS, nos coelhos, adotou-se um modelo baseado em estudos previamente publicados (Burgalassi et al., 1999; El-Shazly et al., 2008), utilizando-se sulfato de atropina colírio $1 \%$, três vezes ao dia, até que se 
confirmasse o diagnóstico de CCS (TLS $\leq 5 \mathrm{~mm} / \mathrm{min}$ e/ou TRB positivo) e durante todo o período de tratamento (12 semanas), para manutenção da CCS. Os momentos de avaliação foram divididos em M0 (antes da indução de CCS), M1 (uma semana após a indução da CCS e início do tratamento) e M2 até M12 (avaliações com intervalo de uma semana após o início do tratamento). Dez coelhos foram induzidos para a CCS conforme o protocolo já descrito e cinco foram alocados no grupo controle, sem indução da CCS e tratados com placebo.

Uma semana após a indução da CCS, os animais foram tratados durante 12 semanas da seguinte maneira: grupo $\mathrm{C}(\mathrm{n}=5)$ controle (uma gota de placebo - solução de $\mathrm{NaCl} 0,9 \%$ ) tópica, duas vezes ao dia, em ambos os olhos; grupo AAP $(n=5)$ : formulação oftálmica com $3,5 \mathrm{~mL}$ de álcool polivinílico $1,4 \%+3,5 \mathrm{~mL}$ de acetilcisteína $10 \%+1 \mathrm{~mL}$ de pilocarpina $1 \%$ (Laboratório Ophthalmos), 1 gota, duas vezes ao dia, por via tópica, em ambos os olhos; grupo L $(n=5)$ : colírio à base de óleo de semente de linhaça puro (Laboratório Ophthalmos), uma gota, duas vezes ao dia, por via tópica, em ambos os olhos.

Semanalmente, os animais foram avaliados com os seguintes exames: avaliação dos sinais clínicos oculares, TLS, TF e TRB. O exame ocular foi realizado com lâmpada de fenda portátil e identificaram-se hiperemia conjuntival, secreção mucoide ou mucopurulenta, opacidade corneal, vascularização ou pigmentação corneal, com os seguintes escores: (0) nenhuma alteração; (1) leve; (2) moderada; (3) severa. O TLS foi realizado sem uso de colírio anestésico para avaliar a quantidade de lágrima produzida. Após limpeza ocular com algodão seco, introduziu-se $0,5 \mathrm{~mm}$ da ponta da fita de Schirmer no saco conjuntival por um período de um minuto, a qual, posteriormente, foi retirada, sendo, em seguida realizada a leitura. Consideraram-se positivos para CCS valores $\leq 5 \mathrm{~mm} / \mathrm{min}$. O TF foi realizado para observar a presença ou não de úlceras de córnea, com a fita de fluoresceína $1 \%$ sendo encostada no saco conjuntival. Utilizaram-se escore 1: negativo para úlcera de córnea; 2: positivo para úlcera de córnea. O TRB foi realizado para a coloração de células desvitalizadas pela CCS. Após instilação de colírio anestésico, foi utilizada uma gota do colírio de Rosa Bengala 0,5\%, considerando-se 1: ausência de células coradas desvitalizadas para CCS; 2: presença de células coradas desvitalizadas para CCS (Maggs, 2008).

A citologia esfoliativa (CE) ocular foi realizada uma vez por mês (M0; M1; M4; M8; M12). Os animais receberam colírio anestésico e, então, foram colhidas amostras da conjuntiva superior, inferior, lateral, medial e córnea de ambos os olhos. A CE foi realizada com o uso de swab umedecido em solução fisiológica e transferida para as lâminas microscópicas. Em seguida, as lâminas foram fixadas em álcool metílico e, posteriormente, coradas pela técnica de MayGrunwald-Giemsa (Raskin e Meyer, 2001).

Para análise histopatológica, ao final do experimento, no M12, foi realizada eutanásia dos coelhos, utilizando-se tiopental sódico a 2,5\%, na dose de $200 \mathrm{mg} / \mathrm{kg}$ por via intravenosa (Neves et al., 2013), para obtenção de biópsia dos olhos. O bulbo ocular, após a enucleação transpalpebral, foi colocado em solução de formalina tamponada a $10 \%$, por 24 a 48 horas. Em seguida, foi lavado em água corrente por 30 minutos, armazenado em álcool $70 \%$ e processado conforme a técnica de inclusão em parafina. Foram obtidos cortes de $5 \mu \mathrm{m}$ de espessura da córnea e conjuntiva, os quais foram corados pela técnica de hematoxilina e eosina (HE) e ácido periódico de Schiff (PAS) (Samuelson, 2007).

Para as variáveis TLS e densidade de células caliciformes, foi utilizado o teste de análise de variância para amostras pareadas, com contrastes pelo método de Tukey. Nos testes acima citados, os pressupostos de normalidade dos dados e de homogeneidade de variâncias foram validados, respectivamente, pelo teste de Shapiro-Wilk e Levene. Foi adotado o nível de significância de $5 \%$.

\section{RESULTADOS}

O tempo de indução da CCS em coelhos foi, em média, de sete a 10 dias. Os sinais clínicos observados no início (M1), no meio (M6) e no final do experimento (M12) estão descritos na Tab. 1. Houve uma melhora significativa dos sinais de hiperemia conjuntival, secreção ocular, opacidade e vascularização corneal do M1 até o M12 nos grupos AAP e L. 
Tabela 1. Médias e desvios-padrão dos valores obtidos* dos sinais clínicos observados em 10 olhos de coelhos experimentalmente induzidos para CCS em cada grupo e submetidos a diferentes tipos de tratamentos: placebo (grupo C); fórmula tópica oftálmica composta por 3,5mL de álcool polivinílico 1,4\% $+3,5 \mathrm{~mL}$ de acetilcisteína $10 \%+1 \mathrm{~mL}$ de pilocarpina $1 \%$ (grupo AAP) e óleo de linhaça tópico (grupo L) nos momentos M1, M6 e M12.

\begin{tabular}{cccccccccc}
\hline Sintomas & \multicolumn{3}{c}{ M1 } & \multicolumn{9}{c}{ M6 } & \multicolumn{3}{c}{ M12 } \\
\cline { 2 - 9 } & $\mathrm{C}$ & $\mathrm{AAP}$ & $\mathrm{L}$ & $\mathrm{C}$ & $\mathrm{AAP}$ & $\mathrm{L}$ & $\mathrm{C}$ & $\mathrm{AAP}$ & $\mathrm{L}$ \\
\hline $\begin{array}{c}\text { Hiperemia } \\
\text { conjuntival }\end{array}$ & $0,0 \pm 0,0$ & $2,0 \pm 0,7$ & $2,0 \pm 0,5$ & $0,0 \pm 0,0$ & $1,0 \pm 0,5$ & $1,0 \pm 0,5$ & $0,0 \pm 0,0$ & $0,5 \pm 0,5$ & $0,5 \pm 0,5$ \\
\hline $\begin{array}{c}\text { Secreção } \\
\text { mucoide ou } \\
\text { purulenta }\end{array}$ & $0,0 \pm 0,0$ & $2,0 \pm 0,5$ & $2,0 \pm 0,5$ & $0,0 \pm 0,0$ & $0,0 \pm 0,5$ & $0,0 \pm 0,0$ & $0,0 \pm 0,0$ & $0,0 \pm 0,0$ & $0,0 \pm 0,0$ \\
$\begin{array}{c}\text { Opacidade } \\
\text { corneal }\end{array}$ & $0,0 \pm 0,0$ & $1,0 \pm 0,7$ & $1,0 \pm 0,5$ & $0,0 \pm 0,0$ & $0,5 \pm 0,5$ & $0,0 \pm 0,5$ & $0,0 \pm 0,0$ & $0,0 \pm 0,5$ & $0,0 \pm 0,0$ \\
$\begin{array}{c}\text { Vascularização } \\
\text { ou pigmentação } \\
\text { corneal }\end{array}$ & $0,0 \pm 0,0$ & $0,5 \pm 0,5$ & $0,0 \pm 0,0$ & $0,0 \pm 0,0$ & $0,0 \pm 0,0$ & $0,0 \pm 0,0$ & $0,0 \pm 0,0$ & $0,0 \pm 0,0$ & $0,0 \pm 0,0$ \\
\hline
\end{tabular}

*(0) nenhuma alteração; (1) leve; (2) moderada; (3) severa,

O grupo $\mathrm{C}$ manteve os parâmetros normais durante o período experimental em todos os testes realizados. A partir do $\mathrm{M} 2$, todos os grupos de tratamento apresentaram aumento estatisticamente significativo $(P<0,05)$ nos valores de TLS (Fig. 1) em comparação com o M1, principalmente o grupo AAP nos momentos finais do experimento (M8 e M10 a M12).

O grupo AAP apresentou animais positivos para o TF (Fig. 2) desde o M3 até o M9, com maior número de animais positivos entre o M4 e o M8. A partir do M9, o grupo AAP apresentou uma resolução das úlceras e permaneceu negativo até o M12. O grupo L foi positivo para TF no M3 e no M4. As úlceras foram todas de grau superficial (Fig. 3A). Tanto o grupo AAP quanto o grupo L foram positivos para o TRB (Fig. 2), a partir do M1, e a maioria corou mais de um quadrante da córnea (Fig. 3B). No grupo AAP, a ausência de células desvitalizadas se deu a partir do M10. Já o grupo L apresentou TRB negativo em $100 \%$ dos animais estudados, a partir do M7.

Em relação ao parâmetro celularidade observado na $\mathrm{CE}$, o grupo AAP apresentou valores moderados a leve em todos os momentos, porém alguns coelhos apresentaram valores acentuados, que foram observados no grupo AAP no M1, M4 e M8, e no grupo L apenas no M12. Em relação ao tipo de célula, tanto o grupo AAP quanto o grupo L apresentaram principalmente células superficiais com apenas alguns coelhos apresentando células parabasais, não se diferenciando dos valores do grupo C (Fig. 4A e 4B). Também foi observada uma presença pequena de células caliciformes na CE (Fig. 4C).

No exame histopatológico em relação à córnea, os grupos L e AAP apresentaram edema leve (Fig. 5A) a moderado (Fig. 5B). No parâmetro degeneração, somente um animal do grupo L apresentou lesão leve. No parâmetro necrose, nenhum grupo apresentou lesão. As células inflamatórias mistas foram constatadas com uma presença moderada no grupo AAP. Na conjuntiva, os grupos AAP e L apresentaram edema leve, degeneração leve no grupo L e ausência de lesão no grupo AAP. Em relação às células inflamatórias, os grupos L e AAP apresentaram uma moderada presença de células mistas, que foram observadas com predomínio na inserção da córnea com a conjuntiva bulbar. Houve um aumento significativo $(P<0,05)$ no número de células caliciformes na conjuntiva no grupo L $(11,9 \pm 2,3)$ e AAP $(12,6 \pm 4,6)$ em relação ao grupo $C(9,8 \pm 1,9)$, e este aumento foi um pouco maior no grupo AAP. 


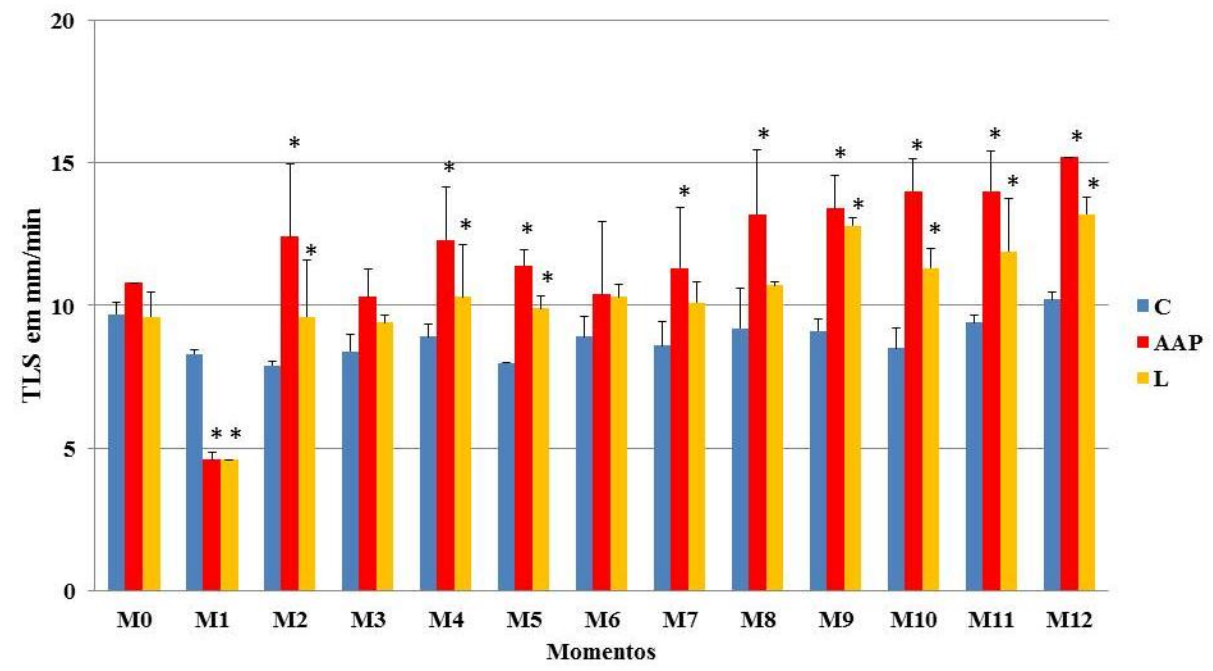

Figura 1. Médias e desvios-padrão dos valores obtidos ${ }^{\mathrm{a}}$ do teste lacrimal de Schirmer (TLS) em mm/min em 10 olhos de coelhos experimentalmente induzidos para CCS em cada grupo e submetidos a diferentes tipos de tratamentos: placebo (grupo C); fórmula tópica oftálmica composta por $3,5 \mathrm{~mL}$ de álcool polivinílico $1,4 \%+3,5 \mathrm{~mL}$ de acetilcisteína 10\% + 1mL de pilocarpina 1\% (grupo AAP) e óleo de linhaça tópico (grupo L) antes (M0) e nas 12 semanas do estudo (M1 a M12).

${ }^{\mathrm{a}}$ valores $\leq 5 \mathrm{~mm} / \mathrm{min}=$ positivo para CCS.

$* P<0.05$ (Teste Tukey).
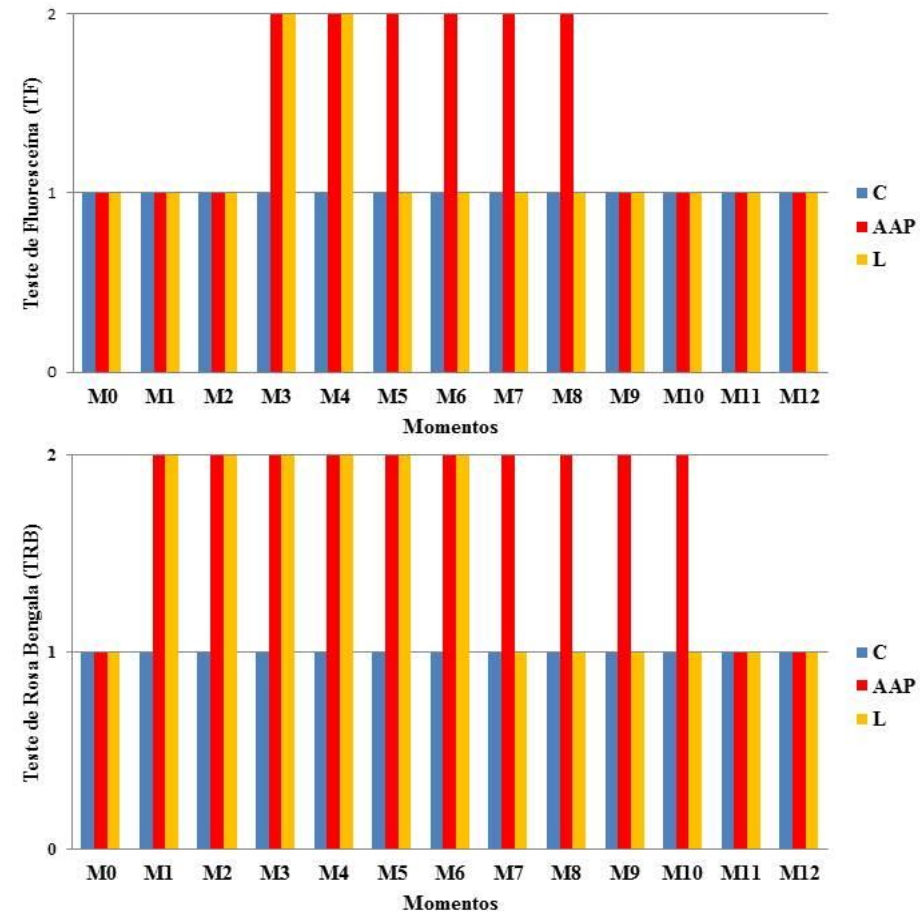

Figura 2. Medianas dos valores obtidos do teste de fluoresceína (TF) ${ }^{\mathrm{a}}$ e do teste de Rosa Bengala (TRB) ${ }^{\mathrm{b}}$ em 10 olhos de coelhos experimentalmente induzidos para CCS em cada grupo e submetidos a diferentes tipos de tratamentos: placebo (grupo C); fórmula tópica oftálmica composta por $3,5 \mathrm{~mL}$ de álcool polivinílico $1,4 \%+3,5 \mathrm{~mL}$ de acetilcisteína $10 \%+1 \mathrm{~mL}$ de pilocarpina $1 \%$ (grupo AAP) e óleo de linhaça tópico (grupo L) antes (M0) e nas 12 semanas do estudo (M1 a M12).

${ }^{a} 1=$ negativo (ausência de úlcera de córnea) e 2=positivo (presença de úlcera de córnea).

${ }^{\mathrm{b}} 1=$ negativo (ausência de células coradas desvitalizadas pela CCS) e 2=positivo (presença de células coradas desvitalizadas pela CCS). 


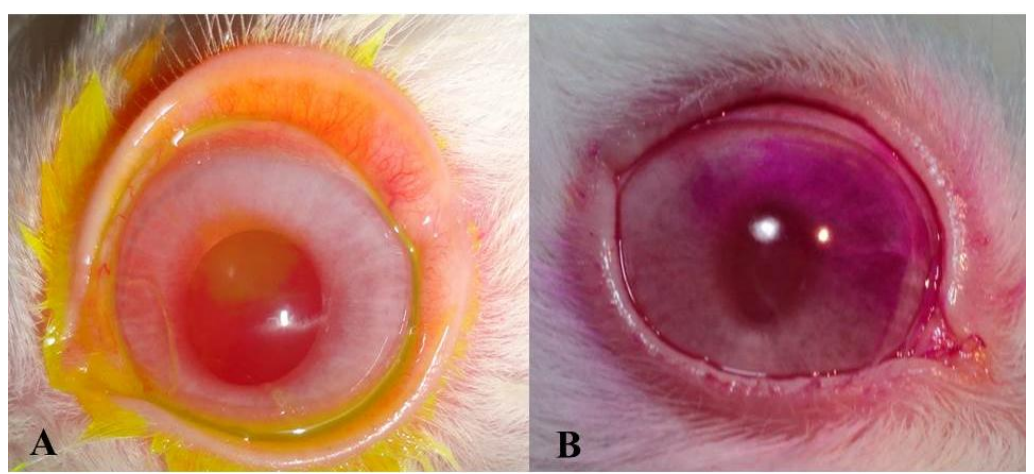

Figura 3. (A) TF positivo demonstrando uma úlcera central superficial e hiperemia conjuntival do coelho n.2 do grupo L no M3. (B) TRB positivo no quadrante superior corneal do coelho n.4 do grupo AAP no M4.
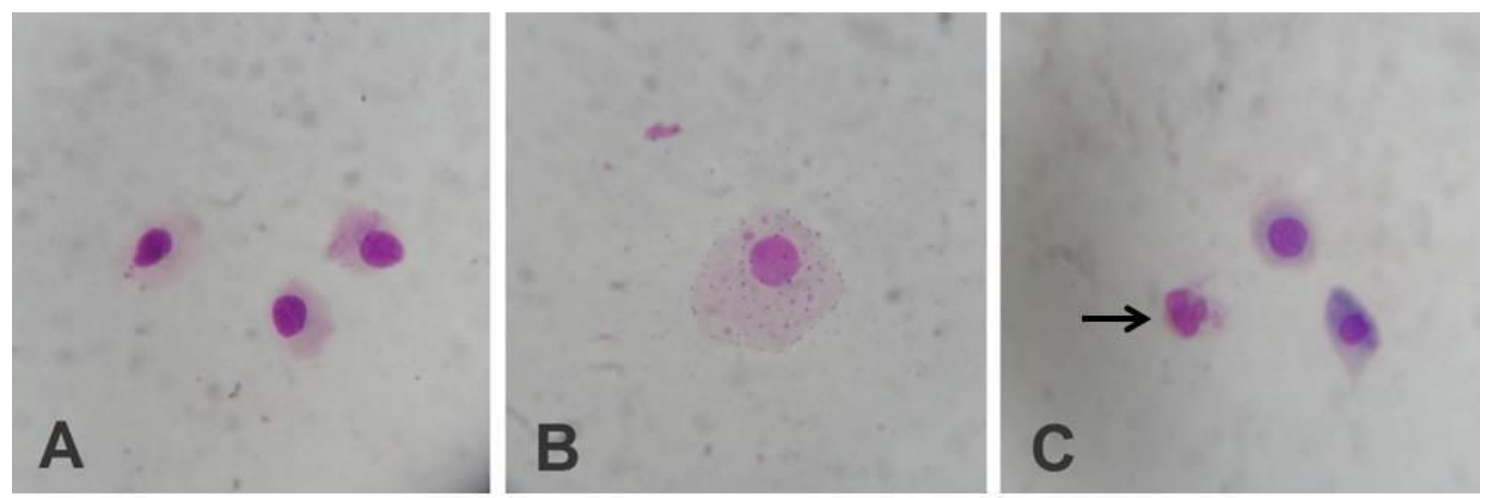

Figura 4. Fotomicrografia de citologia esfoliativa de córnea de coelho. (A) Coelho $\mathrm{n}^{\circ} 3$ do grupo AAP no M1 com presença de células parabasais. (B) Coelho $\mathrm{n}^{\circ} 3$ do grupo AAP no M1 com presença de células superficiais. (C) Coelho $\mathrm{n}^{\circ} 3$ do grupo AAP no momentoM4 com presença de células caliciformes (seta) - May-Grunwald-Giemsa (MGG) (400x).
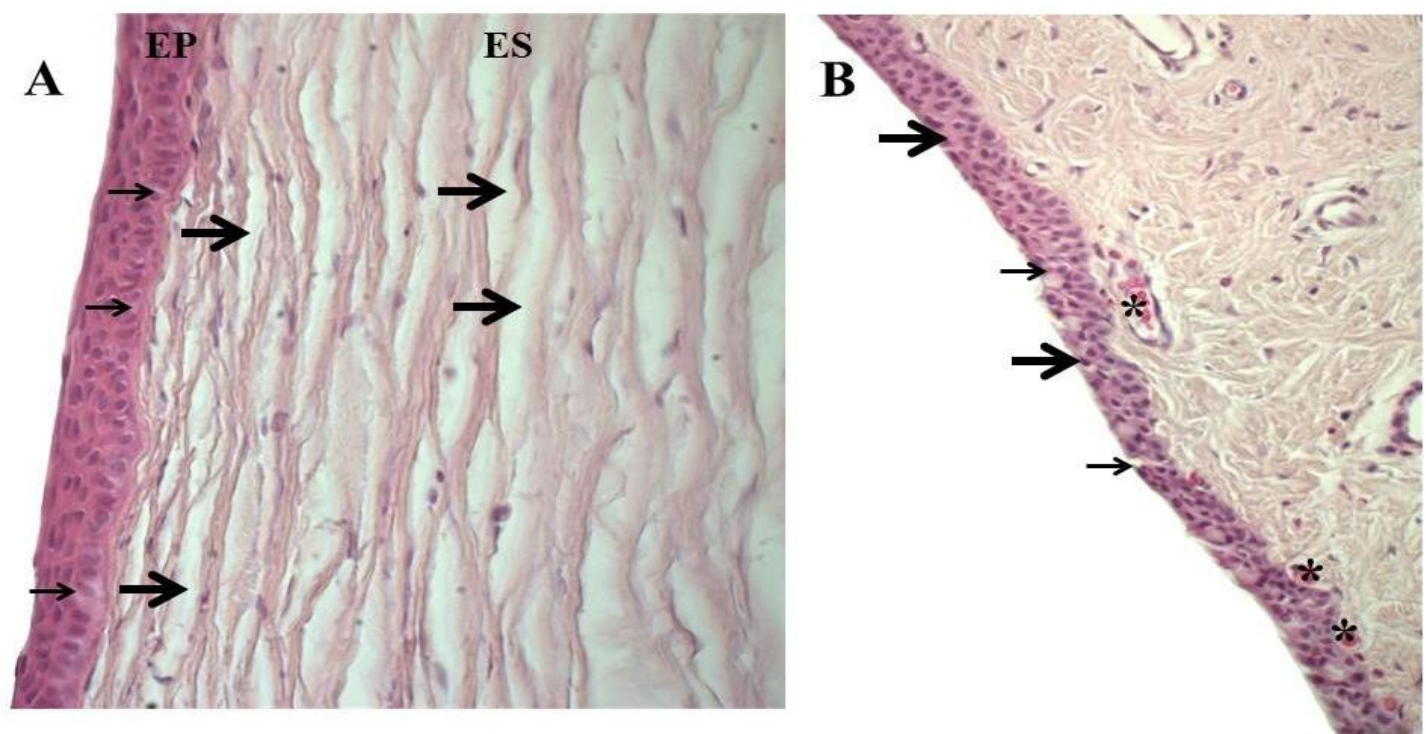

Figura 5. (A) Fotomicrografia de corte de córnea (OE) de coelho $\mathrm{n}^{\mathrm{o}} 3$ do grupo AAP evidenciando edema leve no epitélio corneal (EC) (setas finas) e edema moderado no estroma (E) (setas) - HE (100x). (B) Fotomicrografia da inserção da córnea com a conjuntiva (seta) do coelho $\mathrm{n}^{\circ} 2$ do grupo AAP, onde se observam as células caliciformes (seta). Presença de edema moderado em epitélio corneal (EC) (setas finas). Observa-se congestão de vasos sanguíneos em estroma (E) $(*)$ - HE (400x). 


\section{DISCUSSÃO E CONCLUSÃO}

O uso tópico da formulação oftálmica e do óleo de linhaça rico em ômega 3 e 6 melhorou os sintomas associados à CCS com redução significativa da ocorrência de hiperemia conjuntival, secreção ocular e opacidade corneal, o que está de acordo com os relatos sobre o tratamento da CCS com melhora dos sinais clínicos com a formulação oftálmica tópica em cães (Kirk, 1984; Severin, 1995; Pickett, 2001; Andrade, 2008) e do uso de ômega 3 e 6 em animais de laboratório por via tópica (Rashid et al., 2008; Neves et al., 2013) e em humanos por via oral (Roncone et al., 2010; Pinheiro Jr. et al., 2007; Wojtowicz et al., 2011).

O grupo que utilizou o óleo de linhaça tópico (grupo L) apresentou melhor desempenho na resolução das úlceras de córnea e na diminuição de presença de células desvitalizadas marcadas pelo TRB. O uso tópico de ômega 3 e 6 melhorou os sinais clínicos e inflamatórios induzidos pela CCS (Rashid et al., 2008; Neves et al., 2013) por ser uma fonte natural de mediadores anti-inflamatórios $\omega-6$, prostaglandina-1(PG1) e tromboxano A1 (TXA1), e $\omega-3$, ácido alfalinolênico (ALA), ácido eicosapentaenoico (EPA) e ácido docosahexaenoico (DHA) (Ohma, 2001; Barabino et al., 2003; Jiucheng e Bazan, 2010).

Em se tratando da produção lacrimal, o grupo com formulação oftálmica (grupo AAP) apresentou melhor efeito com relação ao aumento da produção lacrimal, que está relacionada pela associação dos vários componentes integrantes dessa formulação, como o álcool polivinílico a $1,4 \%$, agente umectante que estabiliza a película lacrimal e impede sua ruptura, incrementando a viscosidade (Miller, 2008), a acetilcisteína, que possui propriedade mucolítica, anticolagenolítica e antioxidante (Yalçin et al., 2002; Aldavood et al., 2003; Hongyok et al., 2009), e a pilocarpina, droga parassimpatomimética, que possui efeito lacrimoestimulante, porém pode induzir irritação ocular, caracterizada por hiperemia conjuntival e blefaroespasmo, que não foi observado no presente experimento, talvez devido à sua diluição na formulação oftálmica (Andrade, 2008). Em um estudo em cães, foi relatado que a pilocarpina colírio em várias concentrações $(0,25 ; 1,0$ ou $2,0 \%)$ não alterou significativamente a produção lacrimal, porém a frequência de instilação realizada neste estudo foi nos dias quatro, seis e oito, o que pode ser considerada baixa e ser responsável pelos resultados obtidos (Smith et al., 1994).

$\mathrm{Na} \mathrm{CE}$, não foram observadas diferenças importantes entre os grupos. Nos achados histopatológicos, houve um aumento significativo no número de células caliciformes no grupo L e, principalmente, no grupo AAP em relação ao grupo controle. Este achado é interessante e colabora com a melhora na produção lacrimal e nos sintomas da CCS, visto que a diminuição da quantidade de células caliciformes pode induzir deficiência de mucina no filme pré-corneal e resultar em córneas secas com superfície irregular (Davidson e Kuonen, 2004).

Os resultados demonstraram que houve um aumento na produção lacrimal quando utilizada a formulação oftálmica, uma resolução mais rápida das úlceras de córnea e uma diminuição no número de células desvitalizadas quando utilizado o óleo de semente de linhaça, além de aumento no número de células caliciformes em ambos os grupos de tratamento da CCS induzida em coelhos. Desta maneira, a associação desses dois protocolos pode ser, no futuro, uma alternativa interessante e barata no tratamento da CCS.

\section{AGRADECIMENTOS}

Ao programa de Pós-Graduação em Ciência Animal da Unoeste e à Fapesp, pelo apoio financeiro; ao laboratório Ophthalmos pela doação dos materiais necessários para a execução do experimento.

\section{REFERÊNCIAS}

ALDAVOOD, S.J.; BEHYAR, R.; SARCHAHI, A.A. et al. Effect of acetylcysteine on experimental corneal wounds in dogs. Ophthalmic Res., v.35, p.319-323, 2003

ANDRADE, S.F. Terapêutica Oftálmica. In: ANDRADE S.F. (Ed). Manual de terapêutica veterinária. 3.ed. São Paulo: Roca, 2008. p.179-189.

BARABINO, S.; ROLANDO, M.; CAMICIONE, P. et al. Systemic linoleic and g-linolenic acid therapy in dry eye syndrome with an inflammatory component. Cornea. v.22, p.97-101, 2003. 
BERDOULAY, Y.A.; ENGLISH, R.V.; NALDELSTEIN, B. Effect of topical 0,02\% tacrolimus aqueous suspension on tear productin in dog with keratoconjunctitis sicca. Vet. Opthalmol. v.8, p.225-232, 2005

BURGALASSI, S.; PANICHI, L.; CHETONI P. Development of a simple dry eye model in the albino rabbit and evaluation of some tear substitutes. Ophthalmic Res., v.31, p.229-235, 1999.

CARTER, R.; COLITZ, C.M.H. The causes, diagnosis, and treatment of canine keratoconjunctivitis sicca. Vet Med., v.97, p.683-694, 2002.

DAVIDSON, H.J.; KUONEN, V.J. The tear film and ocular mucin (Review). Vet. Ophthalmol., v.7, p.7177, 2004.

EL-SHAZLY, A.H.; EL-GOHRARY, A.H.; ELSHAZLY, L.H. et al. Comparison between two ciclooxigenase inhibitors in an experimental dry eye model in albino rabbits. Acta Pharma, v.58, p.163173, 2008.

HASSAN-ZADEH, A.; SAHARI, M.A.; BARZEGAR, M. Optimization of the -3 extraction as a functional food from flaxseed. Int. J. Food Sci. Nutrit., v.59, p.526-534, 2008.

HONGYOK, T.; CHAE, J.J.; SHIN, Y.J. et al. Effect of Chitosan-N-Acetylcysteine Conjugate in a Mouse Model of Botulinum Toxin B-Induced Dry Eye. Arch. Ophthalmol., v.127, p.525-532, 2009.

IZCI, C.; CELIK, I.; ALKAN, F. et al. Histologic characteristics and local cellular immunity of the gland of the third eyelid after topical ophthalmic administration of $2 \%$ cyclosporine for treatment of dogs with keratoconjunctivitis sicca. Am. J. Vet. Res., v.63, p.688-694, 2002.

JIUCHENG, H.; BAZAN, H.E.P. Omega-3 fatty acids in dry eye and corneal nerve regeneration after refractive surgery. Prostaglandins Leukot. Essent. Fatty Acids. v.82, p.319-325, 2010.

KIRK, R.W. Atualização Terapêutica Veterinária de Pequenos Animais. São Paulo:Ed Manole, 1984. 1495p.

MAGGS, D.J. Basic diagnostic techiniques. In: MAGGS, D.J.; MILLER, P.E.; OFRI, R. (Ed). Slater's Fundamentas of Veterinary Ophthalmology. 4.ed. St Louis: Elsevier, 2008. p.81-106.

MAVRAGANI, C.P.; MOUTSOPOULOS, N.M.; MOUTSOPOULOS, H.M. The manegement of Söjgren's syndrome. Nat. Clin. Pract. Rheumathol., v.2, p.252-261, 2006.

MILLER, P.E. Lacrimal system. In: MAGGS, D.J.; MILLER, P.E.; OFRI, R. (Ed). Slater's Fundamentas of Veterinary Ophthalmology. 4.ed. St Louis: Elsevier, 2008. p.157-174.
NEVES, M.L.; YAMASAKI, L.; SANCHES, O.C. et $a l$. Use of linseed oil to treat experimentally induced keratoconjunctivitis sicca in rabbits. J. Ophthalmol. Inflamm. Infect., v.3:4, p.1-5, 2013.

OFRI, R.; LAMBROU, G.N.; ALLGOEWER, I. et al. Clinical evaluation of pimecrolimus eye drops for treatment of canine keratoconjunctivitis sicca: a comparison with cyclosporine A. Vet. J., v.179, p.7076, 2009.

OOMAH, B.D. Flaxseed as functional source. J. Sci. Food Agricult., v.81, p.889-894, 2001.

PICKETT, J.P. Up date on veterinary ophthalmology. Ophthalmology Virginia-Maryland Regional College of Veterinary Medicine, 2001. Disponível em: $<$ http://www.dcavm.org/01sep.htm>. Acessado em: 2 nov. 2010.

PINHEIRO JR, M.N.; SANTOS, P.M.; SANTOS, R.C.R. et al. Uso oral do óleo de linhaça (Linum usitatissimum) no tratamento do olho seco de pacientes portadores da síndrome de Sjögren. Arq. Bras. Oftalmol., v.70, p.649-655, 2007.

RASHID, S.; JIN, Y; ECOIFFER, T. et al. Topical omega-3 and omega- 6 fatty acids for treatment of dry eye. Arch. Ophthalmol., v.126, p.210-225, 2008.

RASKIN, R.E; MEYER, D.J. Atlas of canine and feline cytology. WB Saunders: Philadelphia, 2001, 430p.

RONCONE, M.; BARTLETT, H.; EPERJESI, F. Essential fatty acids for dry eye: A review. Contact Lens \& Anterior Eye, v.33, p.49-54, 2010.

SAMUELSON, D.A. Textbook of Veterinary Histology. Saunders Elsevier:St. Louis, 2007, 546p.

SEVERIN, G.A. Severin's Veterinary Ophthalmology Notes. 3. ed. Severin Press: Fort Collins, 1995. 345p.

SMITH, E.M.; BUYUKMIHCI, N.C.; FARVER, T.B. Effect of topical pilocarpine treatment on tear production in dogs. J. Vet. Med. Assoc., v.205, p.12861289,1994

STEVENSON, W.; CHAUHAN, S.K.; DANA, R. Dry eye disease: an immune-mediated ocular surface disorder. Arch. Ophthalmol., v.130, p.130:90-100, 2012.

WOJTOWICZ, J.C.; BUTOVICH, I.; UCHIYAMA, E. et al. Pilot, prospective, randomized, doublemasked, placebo-controlled clinical trial of an omega3 supplement for dry eye. Cornea, v.30, p.308-314, 2011.

YALÇIN, E.; ALTIN, F.; CINHUSEYINOGLUE, F. et al. $\mathrm{N}$-acetylcisteine in chronic blepharitis. Cornea, v.21, p.164-168, 2002. 